Originally published in Physical review E: statistical, nonlinear, and soft matter physics. Vol. 92 No. 3, 2015. http://journals.aps.org/pre/pdf/10.1103/PhysRevE.92.032402

\title{
Proton transfer in liquid water confined inside graphene slabs
}

\author{
Amani Tahat, Jordi Martí* \\ Department of Physics and Nuclear Engineering, \\ Technical University of Catalonia-Barcelona Tech, \\ Building B5, Northern Campus UPC. \\ Jordi Girona, 1-3. 08034 Barcelona. Catalonia. Spain.
}

(Dated: July 28, 2015)

\begin{abstract}
The microscopic structure and dynamics of an excess proton in water constrained in narrow graphene slabs between 0.7 and $3.1 \mathrm{~nm}$ wide has been studied by means of a series of molecular dynamics simulations. Interaction of water and carbon with the proton species was modelled using a multistate empirical valence bond Hamiltonian model. The analysis of the effects of confinement on proton solvation structure and on its dynamical properties has been considered for varying densities. The system is organized in one interfacial and a bulk-like region, both of variable size. In the widest interplate separations, the lone proton shows a marked tendency to place itself in the bulk phase of the system, due to the repulsive interaction with the carbon atoms. However, as the system is compressed and the proton is forced to move to the vicinity of graphene walls it moves closer to the interface, producing a neat enhancement of the local structure. We found a marked slowdown of proton transfer when the separation of the two graphene plates is reduced. In the case of lowest distances between graphene plates $(0.7$ and $0.9 \mathrm{~nm})$, only one-two water layers persist and the two-dimensional character of water structure becomes evident. By means of spectroscopical analysis, we observed the persistence of Zundel and Eigen structures in all cases, although at low interplate separations a signature frequency band around $2500 \mathrm{~cm}^{-1}$ suffers a blue-shift and moves to characteristical values of asymmetric hydronium ion vibrations, indicating some unstability of the typical Zundel-Eigen moieties and their eventual conversion to a single hydronium species solvated by water.
\end{abstract}

PACS numbers: 68.08.De, 61.30.Hn, 61.20.Qg, 82.30.Fi, 31.15.xv

* e-mail: jordi.marti@upc.edu 


\section{INTRODUCTION}

Aqueous proton transfer (PT) is the fundamental process explaining the neutrality of pure water's $\mathrm{pH}[1]$. It is also a fundamental reaction in environments such as in acid-base solutions, in biological systems such as in enzymes or proteins[2] or in electrochemistry, for instance in proton exchange membranes.[3] It also plays a role in basic mechanisms occurring in viruses at the molecular level, such as the human immunodeficiency 1-protease (HIV-1PR).[4] The first study of PT in water[5] was due to von Grotthuss, where it was suggested that the mechanism of PT at normal (ambient) conditions is due to a fast jump between neighboring water molecules. The structure of excess protons in water has been well understood recently,[6] as the result of a series of computer simulations. The general agreement indicates that the aqueous proton can be casted out in terms of a "default" propagating within the three-dimensional network of hydrogen-bonds (HB) of water. This fact produces a disruption of the tetrahedral ordering typical of pure liquid water due to $\operatorname{HBs}[7]$. In this scenario, dynamics of the proton can be regulated by the control of the HB network. Further, since the thermodynamical state of the system determines the main characteristics of the HB network, it also regulates both structure and location of the proton species as well as PT dynamics. At ambient conditions, plenty of information about PT in liquid water and in biomolecular systems at ambient conditions is available.[2, 6, 8-21] Far from ambient conditions, it has been observed that rates of PT and diffusion coefficients of the proton show marked variations from room temperature values, when a wide range of states (from supercooled to supercritical) have been explored.[22-25]

When confined in constrained geometries, the microscopical properties of the proton also suffer drastic changes. So, studies of PT near alumina surfaces[26] and in Nafion fuel cell membranes[27] reported changes in frequencies of vibrational motions and orientational relaxation times induced by the presence of the surface. Recently, a work based on multi-state empirical valence bond (EVB) calculations on PT in one-dimensional water chains confined in carbon nanotubes confirmed early results from Hummer et al.[29] and revealed that the rate of PT inside the tubes was one order of magnitude faster than in bulk.[28] On the other hand, ab-initio molecular dynamics simulations of water inside nanotube channels[29-31] have revealed different mobilities for hydroxide and hydronium ions inside the tubes, depending on the size of the tube and the degree of functionalization of the tube walls. A 
very recent work[32] reports proton transfer within graphene layers when surrounded by water. Protonated water clusters also provide very valuable information to understand proton and water properties at interfaces. First, characteristic signatures of Zundel and Eigen species have been revealed by photoevaporation of weakly bound argon in photofragmentation mass spectroscopy and compared to ab-initio data at MP2 level[33, 34], with reasonably good agreement in most cases. Second, infrared spectroscopy of protonated benzene-water nanoclusters[35] indicated a local ordering of the first water shell around the proton induced by the interface. Very recently, Wang and Agmon[36] have analyzed the balance between dominant structures around the protonated water dimer in water-benzene mixtures, with a clear predominance of a so-called crouching Zundel isomer, symmetrically attached to a benzene ring.

A prototype system to study is that of the aqueous proton confined inside a rectangular slab, changing gradually from a slab containing a large bulk part, up to a system with compressed water forming one or two layers, namely a quasi-two-dimensional water network inside a graphene slab. In a very recent contribution, Bankura and Chandra[37] employed $a b$-initio and quantum-classical simulations to model proton transfer in two-dimensional water layers, in a similar fashion to the present work, fixing the interplate distance to 1.2 nm. In the present paper, we considered variable interplate distances between 3.1 and 0.7 nm. We should expect significant changes in the proton microscopical structure formed (in pure water at ambient conditions) by a series of structures intermediate between two limiting cations: the Zundel dimer $\left(\mathrm{H}_{5} \mathrm{O}_{2}\right)^{+}[38]$ and a hydronium species $\left(\mathrm{H}_{3} \mathrm{O}\right)^{+}$coordinated by water, i.e. the Eigen cation[39] $\left.\left(\mathrm{H}_{9} \mathrm{O}_{4}\right)^{+}\right)$. During computer simulations continuous interconversions between the two structures are usually seen, producing a hybrid $\left(\mathrm{H}_{9} \mathrm{O}_{4}\right)^{+} /\left(\mathrm{H}_{5} \mathrm{O}_{2}\right)^{+}$ complex.[40, 41] The timescale for such interconversions is that of picoseconds, involving changes of oxygen-oxygen (O-O) distances and modifications of the hydrogen connectivity pattern between the hybrid complex at its coordination shells. We also expect significant changes of the time scale of PT due to the presence of confining surfaces and also compared to the case of water inside carbon nanotubes. In summary, the main aim of the present paper is to report a thorough analysis of PT inside a graphene slab by means of molecular dynamics (MD) simulations combined with EVB calculations, paying especial attention to the local structure and dynamical aspects of PT. The technical details will be outlined in Section II, the results of solvation structures of the lone proton and dynamics of PT will be 
described and discussed in Section III and we will give some conclusions in Section IV.

\section{METHOD}

The computer simulations reported in the present work have been performed using a multi-state empirical valence bond approach. The implementation of this methodology to study chemical reactions in solution has been previously described with all sorts of details elsewhere. $[9,10,13,42-54]$ For this reason in the present section we will simply introduce the main features of the method, following the ideas outlined in a previous work[25] together with the specific features implemented for the present contribution. First of all, it is important to note that the account of quantum effects in a simulation may be described in a variety of ways, strongly depending of the characteristics of the system to be analyzed. In the case of fully quantum systems (liquid helium, liquid hydrogen), the choice of methods such as path integral Monte Carlo or ab-initio (Car-Parrinello) molecular dynamics are in order. When the system is composed by a single quantum particle dissolved in a classical bath, semi-classical methods such as the EVB employed in the present work are also very appropriate. Our setup is composed by a quantum particle (excess proton) inside a classical bath (at ambient conditions) formed by 125 flexible TIP3P[55] water molecules. EVB methods assume that the Born-Oppenheimer potential energy surface $\epsilon_{0}(\{\mathbf{R}\})$ driving the dynamics of the nuclei with coordinates $\{\mathbf{R}\}$ can be obtained from the lowest instantaneous eigenvalue of the EVB Hamiltonian:

$$
\hat{\mathrm{H}}_{\mathrm{EVB}}(\{\mathbf{R}\})=\left|\phi_{i}\right\rangle h^{i j}(\{\mathbf{R}\})\left\langle\phi_{j}\right|,
$$

where we have adapted the criterion of summation over repeated indexes. The EVB Hamiltonian is represented in terms of the basis set $\left\{\left|\phi_{i}\right\rangle\right\}$ of diabatic (localized) VB states. In the case of an excess proton in water, these diabatic states are associated to configurations with the $\mathrm{H}^{+}$located in a particular water oxygen. The ground-state $\left|\psi_{0}\right\rangle$ of $\hat{\mathrm{H}}_{\mathrm{EVB}}$ satisfies:

$$
\hat{\mathrm{H}}_{\mathrm{EVB}}\left|\psi_{0}\right\rangle=\epsilon_{0}(\{\mathbf{R}\})\left|\psi_{0}\right\rangle,
$$

and it can be expanded as a linear combination of diabatic states as: 


$$
\left|\psi_{0}\right\rangle=\sum_{i} c_{i}\left|\phi_{i}\right\rangle
$$

leading to the final expression for the potential energy surface:

$$
\epsilon_{0}\{\mathbf{R}\}=c_{i} c_{j} h^{i j}(\{\mathbf{R}\}) .
$$

Dynamics of the nuclei of mass $M_{k}$ is governed by Newton's equation of motion:

$$
M_{k} \frac{\mathrm{d}^{2} \mathbf{R}_{k}}{\mathrm{~d} t^{2}}=-c_{i} c_{j} \nabla_{\mathbf{R}_{k}} h^{i j}(\{\mathbf{R}\}) .
$$

In the framework of EVB methods, off-diagonal elements $h_{i j}$ can be casted out in terms of nuclear coordinates, achieving an excellent agreement with results from full quantum calculations. The parameterization for water and hydronium species employed in the present work follows those proposed by Voth and coworkerset al., $[49,51,53]$ which were applied to different environments and showed excellent agreement with experimental data.

Diagonal elements $h_{i i}$ include contributions from stretching and bending intramolecular interactions within the tagged $\mathrm{H}_{3} \mathrm{O}^{+}$and also inside the rest of water molecules, which are modeled using a flexible TIP3P force field. In addition, diagonal elements also include intermolecular interactions such as those between hydronium-solvent and solvent-solvent. Conversely, off-diagonal elements $h_{i j}$ introduce the coupling between diabatic states $i$ and $j$ and have been modeled including interatomic contributions within a particular $\left(\mathrm{H}_{5} \mathrm{O}_{2}\right)^{+}$ Zundel water dimer spanned by states $\left|\phi_{i}\right\rangle$ and $\left|\phi_{j}\right\rangle$ plus Coulomb interactions between the dimer and the rest of solvent. A complete list of parameters is provided in Ref.[51]. Within this framework, Schmitt et al. were able to reproduce geometries and energies of relevant protonated water clusters $\left(\left(\mathrm{H}_{5} \mathrm{O}_{2}\right)^{+},\left(\mathrm{H}_{7} \mathrm{O}_{3}\right)^{+}\right.$and $\left.\left(\mathrm{H}_{9} \mathrm{O}_{4}\right)^{+}\right)$, obtained from ab initio calculations. Oxygen-carbon and hydrogen-carbon forces were modelled by Lennard-Jones terms with the same parameterization employed in previous works.[56]

The construction of the EVB Hamiltonian was performed as follows:

1. Water molecule closest to the excess proton is located and identified. It constitutes the initial pivot $\mathrm{H}_{3} \mathrm{O}^{+}$and the first diabatic state.

2. From this pivot, the rest of the diabatic states are chosen in a tree-like construction via a HB connectivity pattern. The criterion to establish a HB is as follows: The maximum 
oxygen acceptor-proton donor distance is fixed up to $2.8 \AA$ and the minimum threshold value of the $\mathrm{H}-\mathrm{O}-\mathrm{O}$ angle of $30^{\circ}$.

3. All molecules lying in up to the third solvation shell and showing a connecting path with the original pivot were included in the construction of the $L \times L$ EVB Hamiltonian matrix, which was properly diagonalized.

Typically $L$ is of the order of $\sim 10-20$ units for the connectivity pattern, with fluctuations of total energy always below $1 \%$. At every time step, PT was made possible by re-assigning the pivot oxygen label to the instantaneous state exhibiting the largest $c_{i}^{2}$ coefficient; from this state, the list of participating VB states was reconstructed using the connectivity branching procedure mentioned above. Once the EVB matrix was formed, ground-state eigenvectors and Hellmann-Feynman forces were computed by means of:

$$
\mathbf{F}_{k}=-\left\langle\psi_{0}\left|\frac{\partial \hat{H}_{E V B}}{\partial \mathbf{x}_{k}}\right| \psi_{0}\right\rangle=-c_{i} c_{j} \frac{\partial \hat{H}_{E V B}^{i j}(\mathbf{x})}{\partial \mathbf{x}_{k}} .
$$

In all cases we simulated microcanonical runs at room temperature $T=298 \pm 20 \mathrm{~K}$. In order to maintain the HB network up to some extent we increased density of the system from low (for a $3.1 \mathrm{~nm}$ wide slab) to high values (for a $0.7 \mathrm{~nm}$ wide slab). Slab area was of $3.1 \times 3.1 \mathrm{~nm}$ and excluded space along Z-axis is about $0.3 \mathrm{~nm}$. The simulations were performed with 125 water molecules in all cases. According to the phase diagram of the rigid TIP3P model[57] such conditions would correspond to liquid water states. However, since our model includes flexibility of the molecular bonds the simulated states are only approximately equivalent to the ones of the rigid TIP3P. The pressure in our system will fluctuate, given that we performed our simulations at the microcanonical ensemble (fixed number of particles, volume and energy). As a matter of fact, temperature variations are always small (within 3\%) whereas the range of pressure fluctuations is up to $15 \%$ of the mean value. Our time step was set to $\Delta t=0.5$ fs for all simulations. We considered equilibration periods of 20-30 ps, followed by trajectories of hundreds of ps, used to obtain meaningful statistical properties. Coulombic long-ranged forces were considered by means of Ewald sum techniques,[58] including a uniform neutralizing background. 


\section{RESULTS}

\section{A. Local structure of the excess proton}

We report snapshots of the local proton structure for three different widths of the slab $(d=3.1,1.5$ and $0.7 \mathrm{~nm})$ in Fig.1. We just included, for the sake of clarity, those molecules having the largest weighting coefficients $c_{i}$ from Eq. 4 (22 molecules for $d=3.1 \mathrm{~nm}, 14$ for $d=1.5 \mathrm{~nm}$ and 8 for $d=0.7 \mathrm{~nm})$, which are molecules belonging to the first and second solvation shells of the instantaneous hydronium ("pivot") species. Visual inspection gives a first direct indication on how the local environment of the proton is organized. So, at large interplate distances the proton is found at the central part of the system being the number of diabatic states quite large, very similar to the case of the unconstrained solvated proton (see Ref.[25]). As the graphene plates are placed closer, the number of diabatic states decreases and the number of water layers potentially involved in PT drops to two $(d=1.5 \mathrm{~nm})$ and to roughly one $(d=0.7 \mathrm{~nm})$. The lone proton shows a clear tendency to stay close to the interface, as we will point out with more detail below. In summary, the general structure of the local cluster is dramatically affected by the presence of the graphene plates, which force the system to become quasi-two-dimensional.

To evaluate the location and mobility of the proton species, we computed oxygen pivot $\left(\mathrm{O}^{*}\right)$ z-position at different slab widths, between $d=3.1$ and $0.7 \mathrm{~nm}$, for a time interval of 50 ps. The results are reported in Fig.2. There we observe that in the equilibrated system the lone proton shows a tendency to be transferred in a set of $z$ values ranging from 0.85 to $1.5 \mathrm{~nm}$ for the widest slab $(d=3.1 \mathrm{~nm})$ to values fluctuating around $0.2 \mathrm{~nm}$ for the narrowest case $(d=0.7 \mathrm{~nm})$, where resonant episodes (see below) are hinted. This indicates the tendency of water to mainly diffuse along the $X Y$ plane, when constrained inside a narrow slab. This was already observed for pure water (with no presence of an excess proton) close to a graphene wall[59]. For systems including a lone proton close to hydrophobic interfaces, lateral diffusion of the proton has been observed in membranes made by n-decane molecules[60] together with the well known high-affinity of the proton for the membrane-water interface and also a high proton mobility.

The analysis of the local structure of the proton and of solvating water molecules is made by considering the local pivot-water density profiles: 


$$
\rho_{\mathrm{o}^{*} \alpha}(r)=\frac{1}{4 \pi r^{2}}\left\langle\sum_{i} \delta\left(\left|\mathbf{r}_{\mathrm{o}^{*}}-\mathbf{r}_{i}^{\alpha}\right|-r\right)\right\rangle
$$

where $\mathbf{r}_{\mathrm{O}^{*}}$ is the three-dimensional coordinate of the instantaneous pivot oxygen and $\mathbf{r}_{i}^{\alpha}$ denotes the coordinate of site $(\alpha=\mathrm{O}, \mathrm{H})$ in the $i$-th solvent molecule. Oxygen pivot-oxygen water density profiles are shown in top-left panel in Fig.3. In all cases, the first solvation shell of the proton is centered at $r=2.4 \AA$. This value was already found for unconstrained water at low temperatures[25]. From this information we can observe that the solvent clustering promoted by the proton is strong enough to be barely affected by confinement. In other words, the dynamical equilibrium between Zundel dimer and Eigen cation structures will likely remain in confinement up to a large extent. However, for graphene-graphene distances $d \leq 1.1 \mathrm{~nm}$ the height of the first maxima is reduced. About the second shell around the proton, its center is located at shorter distances as $d$ decreases (about $4 \AA$ instead of 4.5 $\AA$ for the unconstrained case). At shortest distances such as $d=0.9$ and $0.7 \mathrm{~nm}$ marked oscillations of the second shell maxima are seen. This indicates that local clusters tend to become smaller as the two graphene plates become closer. This promotes a larger extent of proton localization in a similar fashion as when proton is at the air-water interface[61].

The analysis of the oxygen pivot-hydrogen water profiles is shown at the bottom panel at left side of Fig.3 and it provides complementary information. Here we found main peaks located at $r=3 \AA$ (first) and $r=5 \AA$ (second) for the unconstrained system, which are reproduced when the interplate distances are over $1.1 \mathrm{~nm}$. The values are in overall agreement with diffraction data obtained by Soper and coworkers[14] for a concentrated $\mathrm{HCl}$ aqueous solution. When $d=1.1 \mathrm{~nm}$ is reached, the position of the second maximum tends to shift backwards, up to be located around $4.75 \AA$ for the case of $d=0.7 \mathrm{~nm}$, favoring higher proton localization. At large slab widths these peaks include exclusively the six hydrogen atoms corresponding the water molecules belonging to the first solvation shell; as width decreases the number of hydrogen atoms included in the second shell tends to be significantly smaller as indicated by short second shell peaks and, especially, by the clear tendency of the first minimum to disappear. This suggests the gradual destruction of the HB network connecting the first and second coordination shells of the lone proton as the interplate distance decreases.

The density profiles of oxygen-oxygen $\rho_{O-O}(r)$ and oxygen-hydrogen $\rho_{O-H}(r)$ (right side 
of Fig. 3) concern the HB connectivity and will have relevance on the mechanisms that drive the transfer of the proton. At the first sight, we can distinguish interfacial and bulk-like regions. The latter become highly fluctuating at low interplate separations (0.7 and $1.1 \mathrm{~nm})$. The locations of the main peaks are in overall good agreement with the findings of Botti et al.[14] for the unconstrained case. As the interplate distance is reduced, maxima corresponding to the second peaks tend to mess up and move to intermediate values, suggesting that as the system is compressed along Z-axis and gradually becomes a quasi-two-dimensional water layer. As described in Ref.[62], the HB network is distorted and eventually broken, at least partially.

\section{B. Dynamics of proton transfer}

As in preliminary works, we will start analyzing the nature of the proton transfer dynamics in the constrained water by direct inspection of the time evolution of the pivot oxygen label during 50 ps time intervals. The results are reported in Fig.4. Representative slab widths $(3.1,2.7,2.3,1.9,1.5,1.1,0.9$ and $0.7 \mathrm{~nm})$ are shown and the effect of confinement on the frequency of proton transfer episodes can be directly observed by simply counting the number of transitions in the figures satisfying that the proton remained attached to a different water molecule for at least $0.5 \mathrm{ps}$ : at $d=3.1 \mathrm{~nm}$ (top panel), approximately $\sim 4$ different water molecules host the proton 0.5 ps or more, roughly delivering a transfer time of the order of $0.1 \mathrm{ps}^{-1}$. That number is about ten times smaller as we move to more compressed water samples. A few PT can be still seen at $d=1.1 \mathrm{~nm}$ and even one of them has been captured at $0.7 \mathrm{~nm}$. We should point out that the predicted rate of transfer at ambient conditions in the bulk, unconstrained system is a factor $\sim 8$ larger than the one inferred from results of NMR experiments[63-65], being this a well know deficiency of the semi-classical picture adopted here; moreover, the explicit incorporation of quantum fluctuations in the transferring proton yields a better agreement with the experiments, leading to rates at least twice as large as the semi-classical ones[49].

Since the jump patterns of the excess proton are simply a sequence of PT episodes, in a large number of cases we may deal with "resonant" episodes where the proton resonates between two valence bond states having large $c_{i}$ coefficients, the so-called "special" bond[48], including aborted transitions, represented by isolated spikes. In order to improve the crude 
picture provided in Fig.4 we need to use time correlation functions. With this aim, equilibrium time correlation functions $C(t)$ for the population relaxation of different reactant species have been considered[53, 66]:

$$
C(t)=\frac{\left\langle\delta h_{i}(t) \cdot \delta h_{i}(0)\right\rangle}{\left\langle\left(\delta h_{i}\right)^{2}\right\rangle}
$$

where the difference $\delta h_{i}(t)=h_{i}(t)-\left\langle h_{i}\right\rangle$ describes instantaneous fluctuations of the population of $i$-th reactant away from its equilibrium value. The characteristic function $h_{i}(t)$ is defined as 1 if the tagged reactant species is present in the system at time $t$ and 0 otherwise. From previous works, we can expect that $C(t)$ defined in eq. 8 will show at least three qualitatively different time domains: (1) a resonant time $\tau_{r s n}$ in the subpicosecond scale, associated to the rapid exchange of the pivot label, i.e. the excess proton, along a "special" bond, represented by spikes in the history of the pivot labels depicted in Fig.4; (2) a second time scale $\tau_{\text {prs }}$ characterizing the averaged lifetime of the resonance episodes and (3) the residence time $\tau_{r s d}$ of the proton when attached to one particular oxygen pivot. Results for the population relaxation of the pivot label are shown in Fig.5. The presence of more than one relaxation time is clear from the absence of a single clear linear regime in the time interval analyzed. By means of Onsager's regression hypothesis[67], we can obtain the proton transfer rates $k_{p}$ from the long time slopes of $C(t)$ (see Fig.5):

$$
k_{p}=\lim _{t \rightarrow \infty}-\frac{\mathrm{d} \ln C(t)}{\mathrm{d} t} .
$$

The average mean residence time of the proton in a pivot water is $\tau_{r s d}=k_{p}^{-1}$. The results for the thermodynamical states considered in this work are reported in Table I.

The general trend is a dramatic slowdown of PT rates when the system is gradually compressed in between the hydrophobic plates, together with the corresponding increase of the estimated residence times $\tau_{r s d}$. The first time interval (that of lifetime of resonant episodes) shows a much faster decay for the unconfined case, whereas the influence of hydrophobic plates is very strong and leads to slowdown of the PT. When comparing to other works, we observe a good overall agreement with findings from Day et al.[53], who obtained a value for the proton transfer rate of $0.3 \mathrm{ps}^{-1}$ at room temperature $(300 \mathrm{~K})$ in the unconstrained case, for an EVB model slightly different of the one used in the present work. In a variety of confined systems, it was observed the preference of the proton to stay at interfaces, where free 
energy minima have been found[31, 60]. This would favor the gradual reduction of PT rates that, in the present case must be influenced by the fact that the pressure has considerably grown due to the increase of the density at short interplate distances. Probably because of this fact proton transfer rates obtained in the present work are significantly smaller than those obtained from ab-initio simulations by Bankura and Chandra[37].

\section{Proton diffusion}

The diffusion coefficient of aqueous protons at ambient conditions is known to be approximately fourfold that observed for neat water. So, the experimental value is of $0.93 \AA^{2} / \mathrm{ps}[68]$ for a proton diffusing in water at $298.15 \mathrm{~K}$ and at the density of $1 \mathrm{gcm}^{-3}$, whereas the value of the diffusion coefficient of bulk liquid water is of $0.23 \AA^{2} / \mathrm{ps}[69]$. The main reason of such an enhancement of the diffusion is well known and is based on the Grotthuss translocation mechanism, [5] in addition to the usual hydrodynamic Stokes mass diffusion. In simulations of excess protons inside quasi-one-dimensional environments, such as carbon nanotubes, it has been observed that proton diffusion is strongly affected by the tube radius and it can be either faster than in bulk water or slower[70]. The threshold is around radii of 7-8 $\AA$. At interfaces, such as in the case of water near n-decane[60], lateral diffusion of the proton has been observed together with a delay of the exchange of protons between the bulk region and the interfacial region.

The calculation of diffusion coefficients of aqueous protons $D_{p}$ in the constrained system considered in the present work reveals interesting changes in this scenario, as it happens at interfaces or at high temperature and in supercritical states[24]. We obtained $D_{p}$ from long time slopes of mean square displacements of the proton coordinate $\mathbf{r}_{p}$, in the usual way:

$$
D_{p}=\frac{1}{6} \lim _{t \rightarrow \infty} \frac{\mathrm{d}}{\mathrm{d} t}\left\langle\left|\mathbf{r}_{p}(t)-\mathbf{r}_{p}(0)\right|^{2}\right\rangle
$$

where the proton coordinate was defined as a weighted sum of the coordinates of the $L$ pivot molecules, $\mathbf{r}_{p v t}^{i}$ :

$$
\mathbf{r}_{p}=\sum_{i}^{L} c_{i}^{2} \mathbf{r}_{p v t}^{i} .
$$

Results for the diffusion coefficients are shown in Fig.6 and numerically reported in Table 
I. Two important features should be discussed: (1) The general trend of the proton mobility is a neat reduction from $0.94 \AA^{2} /$ ps at the unconstrained state to lower values up to one order of magnitude smaller inside the graphene slab (case $d=0.7 \mathrm{~nm}$ ). $D_{p}$ at $298 \mathrm{~K}$ is in overall good agreement with previous works[24] and in excellent agreement with the experimental value reported above (this may be fortuitous). (2) The simulation results also indicate that the reduction of $D_{p}$ for decreasing interplate distances $d$ is roughly linear. Here we should keep in mind that we set up our simulations in such a way that density is bigger at low $d$, so that an important part of the HB network has survived. Since the Grotthuss shuttling operates via HB, the mechanism can work in all cases, even at the quasi-two-dimensional slab at $d=0.7 \mathrm{~nm}$. To compare with a similar system, Bankura and Chandra[37] obtained values around $0.1 \AA^{2} / \mathrm{ps}$ for a graphene slab $1.2 \mathrm{~nm}$ wide, which is quite well matched by the value of $0.23 \AA^{2} / \mathrm{ps}$ reported in this work for the interplate distance of $1.1 \mathrm{~nm}$. The factor two between these works should be due to a density effect, within the relative accuracies and reliabilities of the different methodologies employed.

As it was observed previously[25], the structural characteristics of the local proton environment has significant influence on proton diffusion: at bulk water ambients the proton jumps a characteristic O-O distance, say $d_{\mathrm{OO}} \sim 3.5 \AA$, every $\tau \sim 2$ ps. In this interval, the center of mass of a water molecule travels typically $l \sim\left(6 D_{w} \tau\right)^{1 / 2} \sim 2 \AA$; so $l$ is comparable to $d_{\mathrm{OO}}$ and PT operates safely through Grotthuss mechanism. A similar calculation performed at $d=3.1 \mathrm{~nm}$ gives an average time for PT of approximately $\tau \sim 14$ ps whereas water diffusion is slower $\left(0.67 \AA^{2} / \mathrm{ps}\right.$, see Table I) yielding $l \sim 7.5 \AA$ and showing that spatial displacements during the proton translocation are significantly bigger than those of $d_{\mathrm{O}^{*} \mathrm{O}} \sim 2.5$ $\AA$ (see Fig.3) what makes more difficult the transfer of the proton. Even though our model EVB Hamiltonian is likely to predict a lower rate of proton transfer and a subestimation of the actual diffusion, we do believe that the differences are sufficiently large to guarantee that the qualitative picture captured by our simulations remains physically meaningful.

\section{Proton spectroscopy}

The analysis of microscopic vibrations in water is usually performed with the help of Raman or infrared spectroscopy. Infrared spectra report properties such as the absorption coefficient, $\alpha(\omega)$ or the imaginary part of the dielectric constant, $\varepsilon^{\prime \prime}(\omega)[71]$ which are of 
quantum nature. Such properties can be computed in the EVB framework with the aid of an absorption lineshape function $I(\omega)$, i.e. the Fourier transform of the time derivative of the dipole moment $(\dot{\mu}(t))[48,72]$. In this work, we have considered an alternative observable, namely the velocity autocorrelation function of the lone proton

$$
C_{p}(t)=<\mathbf{v}_{p}(0) \cdot \mathbf{v}_{p}(t)>
$$

where the proton velocity $\mathbf{v}_{p}(t)$ can be obtained directly from the time derivative of its position $\mathbf{r}_{p}$ :

$$
\mathbf{v}_{p}(t)=\frac{\mathrm{d} \mathbf{r}_{p}(t)}{\mathrm{d} t}
$$

From Eq.(12), by means of the usual Fourier transform, we can obtain a vibrational density of states $S_{p}(\omega)[51]$ :

$$
S_{p}(\omega)=\int_{0}^{\infty} \mathrm{d} t C_{p}(t) e^{i \omega t}
$$

With this assignment, we have computed $S_{p}(\omega)$ for all slab widths considered along the present work. The length of $C_{p}(t)$ has been of $0.5 \mathrm{ps}$, long enough to capture all relevant proton vibrations, but also much shorter than the proton residence time (always larger than 10 ps, see Table I). However, as a matter of fact, we will be able to obtain relevant modes of vibration of the hydronium $\mathrm{H}_{3} \mathrm{O}^{+}$complex. The results are shown in Fig.7. We have chosen to show roughly the full frequency range, since although specific spectral signatures of proton vibrations are located between 1400 and 3000 wavenumbers[48, 72], some authors like Hammer et al.[33] include the range between 850 and 1400 as relevant for shared proton motions.

Within the specific region of proton vibrations, three common features in the spectrum $S_{p}(\omega)$ for the unconstrained case (bottom plot, Fig.7, black line) are clearly observed, represented by maxima at: (1) $1445 \mathrm{~cm}^{-1}$, labeled as (A); (2) $2370 \mathrm{~cm}^{-1}$, labeled as (C-D); and (3) $2960 \mathrm{~cm}^{-1}$, labeled as (E). Further, a broad band between $600-1000 \mathrm{~cm}^{-1}$ (F) might be also related to low-frequency proton vibrations. In all computed spectra, the uncertainty in the frequency location of maxima is of the order of $10 \mathrm{~cm}^{-1}$, as it has been obtained from a series of independent simulations. When spectra from the confined proton are considered, some spectral shifts $\Delta \omega$ have been found. So, the band (A) is red-shifted by 50-100 
wavenumbers at the widest slab widths $(d=3.1$ to $1.5 \mathrm{~nm})$, whereas shows a blue-shift of around 75 wavenumbers for the narrowest slab separations, namely 0.7 and $0.9 \mathrm{~nm}$. In the case of band (C-D), all shifts are towards blue but while for the separations between 3.1 and $1.5 \mathrm{~nm}$ the absolute value is $\Delta \omega=30-40 \mathrm{~cm}^{-1}$, at narrow separations it becomes much bigger (around $200 \mathrm{~cm}^{-1}$ ). Finally, the band (E) reveals a neat blue-shift of the order of 200 $\mathrm{cm}^{-1}$ for all cases. The band maxima associated to proton vibrations are in an overall good qualitative agreement with experimental data available. Fourier transform infrared spectra (FTIR) measurements of $\mathrm{HCl}$ and $\mathrm{NaCl}$ aqueous solutions at different concentrations at room temperature[72] revealed maxima associated with hydrated protons at 1200, 1800 and $2900 \mathrm{~cm}^{-1}$ whereas Headrick et al.[34] considered protonated water clusters in argon and reported proton vibrations in a hydronium ion at 2665 and water vibrations inside a Zundel dimer at $3160 \mathrm{~cm}^{-1}$, from photoevaporation of argon in photofragmentation mass spectroscopy. For the same system, Hammer et al.[33] reported bands around 1000-1150 $\mathrm{cm}^{-1}$ that were assigned to shared proton (Zundel dimer) vibrations. In summary, these values are in reasonably good qualitative agreement with the frequencies around 900, 1445 and $2960 \mathrm{~cm}^{-1}$ reported in the present work. In addition, the band (C-D) centered around $2370 \mathrm{~cm}^{-1}$ can be considered in close agreement with the maxima at $2420 \mathrm{~cm}^{-1}$ reported in Ref.[34] associated to a hydronium symmetric stretch mode.

As a benchmark for the force field considered in this work, we computed the the vibrational bands associated with the isolated Zundel dimer and Eigen complex, equivalent to gas phase[25] (see Fig.7). In particular, proton vibrational modes for the Zundel dimer occur at different frequencies, about 1150, label (F) and $1880 \mathrm{~cm}^{-1}$, label $(\mathrm{G})$ than those of the Eigen complex, around 1400 (A), 1750 (B), 2500 (C), 2750 (D) and $3650 \mathrm{~cm}^{-1}$ (E). The agreement of these values with corresponding ones from experimental data is quite satisfactory. On the one hand, Schwartz[73] reported a frequency maximum about $2660 \mathrm{~cm}^{-1}$ for a $\mathrm{H}_{9} \mathrm{O}_{4}^{+}$cluster (Eigen complex) from infrared absorption spectra of several water clusters in gas phase, attributed by Okumura et al.[74] to a hydronium $\left(\mathrm{H}_{3} \mathrm{O}^{+}\right)$ion' stretch. On the other hand, a maximum at about $1740 \mathrm{~cm}^{-1}$ was reported from experimental measurements of the gas-phase infrared spectrum of the protonated water dimer[75] $\left(\mathrm{H}_{5} \mathrm{O}_{2}^{+}\right)$. The reported results from computed vibrational density of states by Schmitt and Voth[51] for a similar potential model were of 1550 and $2860 \mathrm{~cm}^{-1}$ for the two complexes, what indicates again a good agreement with our results. Further, the agreement is reasonably good with 
results from Vuilleumier and Borgis[48] for a flexible SPC/E model, who reported stretching modes of the hydronium complex at 2000 and $2650 \mathrm{~cm}^{-1}$ and with those obtained by Voth and coworkers[72]: these authors assigned the modes around 1680-1880 and 3250-3400 $\mathrm{cm}^{-1}$ to pure Zundel-like vibrations, and the modes around 1580-1640 and 2700-2950 to pure Eigen-like vibrations.

Comparing the reference maxima for Zundel and Eigen moieties (top Fig.7) with the spectral profiles obtained for the condensed liquid system (bottom of Fig.7), we can draw some additional clues: (1) the bands centered at 1445 and $2960 \mathrm{~cm}^{-1}$ in the spectrum of proton in confined water inside the graphene slab (from now on "aqueous proton") match well the maximum (A) for the isolated Eigen complex; (2) the band maximum at 2370 $\mathrm{cm}^{-1}$ can be related to the maxima (C) and (D) of the isolated Eigen and (3) the broad band centered around $750-1000 \mathrm{~cm}^{-1}$ in the spectral densities at the bottom of Fig. 7 have no clear counterpart at the top of the same figure, although the closest band maxima is a peak at $1100 \mathrm{~cm}^{-1}$ of the Zundel power spectrum. In summary, the dynamical exchange between the two species seems to remain in confinement, even in the case of quasi-twodimensional structures formed by a few water layers. As an additional fact, we should note that a vibrational frequency around $2400-2600 \mathrm{~cm}^{-1}$ was reported by Headrick et al.[34] and assigned to the asymmetric stretch motions of hydronium. Following this, the band labeled $(\mathrm{C}-\mathrm{D})$ in the aqueous proton spectrum might indicate the presence of a lone hydronium ion in the states of higher compression $(0.7,0.9 \mathrm{~nm})$ as indicated by the blue-shift of this $(\mathrm{C}-\mathrm{D})$ band as $d$ decreases. This fact that hydronium species may replace Zundel cations (and/or Eigen complexes) was already observed by Habenicht et al.[76] in their study of the effects of hydrophobic confinement on protons from acidic systems.

\section{CONCLUDING REMARKS}

In the present work a thorough analysis of the structure and dynamics of an excess proton in liquid water under confinement inside a slab formed by two parallel graphene plates has been reported. The system was set to tune the water layers from full three dimensional states to states at short interplate distances ( $0.7 \mathrm{~nm}$ wide) where water structure can be regarded as quasi-two-dimensional. We employed MD simulations together with a multidimensional empirical valence bond procedure, in order to construct a suitable Hamiltonian for the semi- 
classical system, formed by a quantum particle (the lone proton) embedded in a sea of classical flexible TIP3P waters.

Our findings have revealed the enhancement of the local structure of the proton in the narrowest pores. At the quasi two-dimensional water states represented by slab's widths of $d=0.7$ and $0.9 \mathrm{~nm}$, the environment of the proton typical of ambient conditions, consisting of a mixture of Zundel and Eigen-like structures has evolved to a network of 2D water molecules, still including Zundel and Eigen complexes but allowing the breaking of these structures in favor of a lighter species, the hydronium ion, as revealed by the existence of suitable frequency shifts towards higher values, as it can be inferred from available spectroscopical data. So, the proton in 2D water would remain trapped to an hydronium complex for quite long time intervals, given the averaged transfer time of more than 200 ps, whereas at unconstrained conditions the mean time for a proton transfer is of the order of 1-2 ps. This indicates that PT still occur, but at much shorter rates of the order of $10^{-3} \mathrm{ps}^{-1}$. Diffusion of the proton tends to decrease moderately when the system is compressed, changing from 0.94 $\AA^{2} /$ ps in unconstrained bulk water up to a factor 4.5 fold smaller at quasi-two-dimensional water, i.e. for $d=0.7$ and $0.9 \mathrm{~nm}$. Our results for diffusion coefficients agree well with those of Bankura and Chandra[37], although proton transfer rates obtained in this work are significantly smaller than those from the same authors, probably due to the higher water density considered here.

The analysis reported in the present paper is a first step into the study of PT in two dimensional systems and it should be completed by the analysis of the system at low and high temperatures, currently under progress in our laboratory.

\section{Acknowledgments}

We thank financial support from the Direcció General de Recerca de la Generalitat de Catalunya (grant 2009SGR1003) and the Spanish Ministry of Economy and Competitiveness (grant FIS2012-39443-C02-01). 
[1] P.L.Geissler, C.Dellago, D.Chandler, J.Hutter and M.Parrinello, Science 291, 2121 (2001).

[2] G.A.Voth, Acc.Chem.Res. 39, 143 (2006).

[3] J.T.Hynes, J.P.Klinman, H.-H.Limbach and R.L.Schowen (eds.), Hydrogen-Transfer reactions (John Wiley and sons, New York, 2006).

[4] J.Trylska, P.Grochowski and J.A.McCammon, Protein Sci. 13, 513 (2004).

[5] C.J.D.von Grotthus, Ann.Chim. LVIII, 54 (1806).

[6] D.Marx, M.E.Tuckerman, J.Hutter and M.Parrinello, Nature 397, 601 (1999).

[7] D.Eisenberg and W.Kauzmann, The structure and properties of water (Clarendon Press, Oxford, 1969).

[8] M.E.Tuckerman, D.Marx, M.L.Klein and M.Parrinello, Science 275, 817 (1997).

[9] T.J.F.Day, U.W.Schmitt and G.A.Voth, J.Am.Chem.Soc. 122, 12027 (2000).

[10] S.Walbran and A.A.Kornyshev, J.Chem.Phys. 114, 10039 (2001).

[11] M.E.Tuckerman, D.Marx and M.Parrinello, Nature 417, 925 (2002).

[12] H.J.Baker and H.-K.Nienhuys, Science 297, 587 (2002).

[13] A.A.Kornyshev, A.M.Kuznetsov, E.Spohr and J.Ultrup, J.Phys.Chem.B 107, 3351 (2003).

[14] A.Botti, F.Bruni, S.Imberti, M.A.Ricci and A.K.Soper, J.Chem.Phys. 121, 7840 (2004).

[15] D.Asthagiri, L.R.Pratt and J.D.Kress, Proc.Natl.Acad.Sci.USA 102, 6704 (2005).

[16] A.Botti, F.Bruni, M.A.Ricci and A.K.Soper, J.Chem.Phys. 125, 014508 (2006).

[17] D.Marx, Chem.Phys.Chem. 7, 1848 (2006).

[18] M.E.Tuckerman, A. Chandra and D.Marx, Acc.Chem.Res. 39, 151, (2006).

[19] J.M.J.Swanson, C.M.Maupin, H.Chen, M.K.Petersen, J.Xu, Y.Wu and G.A.Voth, J.Phys.Chem.B 111, 4300 (2007).

[20] D.Marx, A.Chandra and M.E.Tuckerman, Chem.Rev. 110, 2174, (2010).

[21] S.H.Lee and J.C.Rasaiah, J.Chem.Phys. 135, 124505 (2011).

[22] C.Kobayashi, S.Saito and I.Ohmine, J.Chem.Phys. 113, 9090 (2000).

[23] C.Kobayashi, S.Saito and I.Ohmine, J.Chem.Phys. 115, 4742 (2001).

[24] D.Laria, E.Guàrdia and J.Martí, J.Am.Chem.Soc. 126, 2125 (2004).

[25] A.Tahat, J.Martí, Phys.Rev.E 89, 052130 (2014).

[26] S.Le Caër, D.J.Palmer, M.Lima, J.P.Renault, G.Vigneron, R.Righini and S.Pommeret, 
J.Am.Chem.Soc. 129, 11720 (2009).

[27] D.B.Spry, A.Goun, K.Glusac, D.E.Moilanen and M.D.Fayer, J.Am.Chem.Soc. 129, 8122 (2007).

[28] Z.Cao, Y.Peng, T.Yan, S.Li, A.Li and G. Voth, J.Am.Chem.Soc. 132, 11395 (2010).

[29] G.Hummer, J.C.Rasaiah and J.P.Noworyta, Nature 414, 188 (2001).

[30] A.Bankura and A. Chandra, J.Phys.Chem.B 116, 9744 (2012).

[31] J.K.Clark and S.J.Paddison, Phys.Chem.Chem.Phys. 16, 17756(2014).

[32] J.L.Achtyl, R.R.Unocic, L.Xu, Y.Cai, M. Raju, W.Zhang, R.L.Sacci, I.V.Vlassiouk, P.F.Fulvio, P.Ganesh, D. J.Wesolowski, S.Dai, A.C.T. van Duin, M.Neurock and F.M.Geiger, Nature Comm. 6, 6539 (2015).

[33] N.I.Hammer, E.G.Diken, J.R.Roscioli, M.A.Johnson, E.M.Myshakin, K.D.Jordan, A.B.McCoy, X.Huang, J.M.Bowman, and S.Carter, J.Chem.Phys. 122, 244301 (2005).

[34] J.M.Headrick, E.G.Diken, R.S.Walters, N.I.Hammer, R.A.Christie, J.Cui, E.M.Myshakin, M.A.Duncan, M.A.Johnson and K.D.Jordan, Science, 308, 1765 (2005).

[35] T.C.Cheng, B.Bandyopadhyay, J.D.Mosley and M.A.Duncan, J.Am.Chem.Soc. 134, 13046 (2012).

[36] H.Wang and N.Agmon, J.Phys.Chem.B 119, 2658 (2015).

[37] A.Bankura and A. Chandra, J.Chem.Phys. 142, 044701 (2015).

[38] G.Zundel and H.Metzger, Z.Phys.Chem. 244, 456 (1968).

[39] M.Eigen and L.de Maeyer, Proc.R.Soc.(London), A247, 505 (1958).

[40] M.Tuckerman, K.Laasonen, M.Sprik and M.Parrinello, J.Chem.Phys. 103, 150 (1995).

[41] M.Tuckerman, K.Laasonen, M.Sprik and M.Parrinello, J.Phys.Chem.B 99, 5794 (1995).

[42] C.A.Coulson, U.Danielsson, Ark.Fys. 8, 239 (1954).

[43] R.S.Mulliken, J.Chem.Phys. 61, 20 (1964).

[44] J.Aqvist and A.Warshel, Chem.Rev. 93, 2523 (1993).

[45] A.Warshel, Computer Modeling of Chemical Reactions in Enzymes and Solutions (Wiley, New York, 1980).

[46] R.Vuilleumier and D.Borgis, J.Phys.B. 102, 4261 (1998).

[47] R.Vuilleumier and D.Borgis, Chem.Phys.Lett. 284, 71 (1998).

[48] R.Vuilleumier and D.Borgis, J.Chem.Phys. 111, 4251 (1999).

[49] J.Lobaugh and G.A.Voth, J.Chem.Phys. 104, 2056 (1996). 
[50] U.W.Schmitt and G.A.Voth, J.Phys.Chem.B 102, 5547 (1998).

[51] U.W.Schmitt and G.A.Voth, J.Chem.Phys. 111, 9361 (1999).

[52] M.Cuma, U.W.Schmitt and G.A.Voth, Chem.Phys.Lett. 258, 187 (2000).

[53] T.J.F.Day, A.V.Soudackov, M.Cuma, U.W.Schmitt and G.A.Voth, J.Chem.Phys. 117, 5839 (2002).

[54] D.E.Sagnella and M.E.Tuckerman, J.Chem.Phys. 108, 2073 (1998).

[55] L.X.Dang and B.M.Pettitt, J.Phys.Chem. 91, 3349 (1987).

[56] M.C.Gordillo and J.Martí, Chem.Phys.Lett. 329, 341 (2000).

[57] C.Vega, J.L.F.Abascal, M.M.Conde and J.L.Aragones, Faraday Discuss. 141, 251 (2009).

[58] D.Frenkel and B.Smit, Understanding Molecular Simulation (Academic Press, San Diego, 2002).

[59] J.Martí, G.Nagy, E.Guàrdia and M. C. Gordillo, J.Phys.Chem.B 110, 23987 (2006).

[60] C.Zhang, D.G.Knyzaev,Y.A.Vereshaga, E.Ippoliti, T.H.Nguyen, P.Carloni and P.Pohl, Proc.Natl.Acad.Sci.USA 109, 9744 (2012).

[61] R.Kumar, C.Knight and G.A.Voth, Faraday Discuss. 167, 263 (2013).

[62] M.C.Gordillo, G.Nagy and J.Martí, J.Chem.Phys. 123, 054707 (2005).

[63] S.Meiboom, J.Chem.Phys. 34, 375 (1961).

[64] H.G.Herzt and R.Manner, Z.Phys.Chem.Neue Folge 135, 89 (1983).

[65] R.Pfeifer and H.G.Hertz, Ber.Bunsen-Ges.Phys.Chem. 94, 1349 (1990).

[66] R.Semino and D.Laria, J.Chem.Phys. 136, 194503 (2012).

[67] D.Chandler, Introduction to Modern Statistical Mechanics, (Oxford University Press, New York, 1987).

[68] R.A.Robinson and R.H.Stokes, Electrolyte Solutions (Butterworths, London, 1959).

[69] K.Krynicki, C.D.Green and D.W.Sawyer, Faraday Discus.Chem.Soc. 66, 199 (1978).

[70] M.E.Selvan, D.J.Keffer, S.Cui and S.J.Paddison, Mol.Simul. 36, 568 (2010).

[71] D.A.McQuarrie, Statistical Mechanics (University Science Books, Sausalito, California, 2000).

[72] J.Kim, U.W.Schmitt, J.A.Gruetzmacher, G.A.Voth and N.E.Scherer, J.Chem.Phys. 116, 737 (2002).

[73] H.A.Schwarz, J.Chem.Phys. 67, 5525 (1977).

[74] M.Okumura, L.I.Yeh, J.D.Myers and Y.T.Lee, J.Phys.Chem. 94, 3416 (1990).

[75] K.R.Asmis, N.L.Pivonka, G.Santambrogio, M.Brümmer, C.Kaposta, D.M.Neumark, L.Wöste, 
Science, 299, 1375 (2003).

[76] B.F.Habernicht, S.J.Paddison and M.E.Tuckerman, J.Mater.Chem. 20, 6342 (2010). 
TABLE I: Dynamical parameters for the aqueous protons at different slab widths: proton transfer rates $\tau_{r s d}^{-1}$, residence time $\tau_{r s d}$ and diffusion coefficient of the lone proton $D_{p}$. Data for the unconstrained system at ambient conditions were obtained from Ref.[25].

\begin{tabular}{c|c|c|c}
\hline$d(\mathrm{~nm})$ & $\tau_{r s d}^{-1}\left(\mathrm{ps}^{-1}\right)$ & $\tau_{r s d}(\mathrm{ps})$ & $D_{p}\left(\AA^{2} / \mathrm{ps}\right)$ \\
\hline 3.1 & 0.071 & 14.1 & 0.67 \\
2.7 & 0.053 & 18.9 & 0.57 \\
2.3 & 0.028 & 35.7 & 0.52 \\
1.9 & 0.021 & 47.6 & 0.38 \\
1.5 & 0.011 & 90.9 & 0.33 \\
1.1 & 0.0045 & 222.2 & 0.23 \\
0.9 & 0.0028 & 357.1 & 0.20 \\
0.7 & 0.0011 & 909.1 & 0.18 \\
unconstrained & 0.54 & 1.85 & 0.94 \\
\hline
\end{tabular}

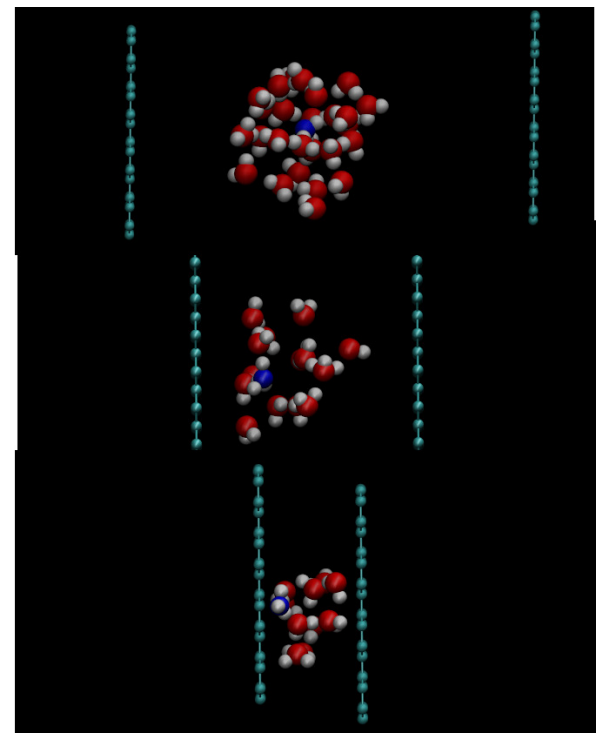

FIG. 1: (Color online) Snapshots of local configurations around the pivot water at different slab widths (top to bottom): $d=3.1,1.5$ and $0.7 \mathrm{~nm}$. Only water molecules having largest coefficients $C_{i}$ (typically 20-30 molecules) are explicitly shown. Carbon atoms (cyan), oxygens (red), hydrogens (white), pivot (see text) oxygen (blue). 


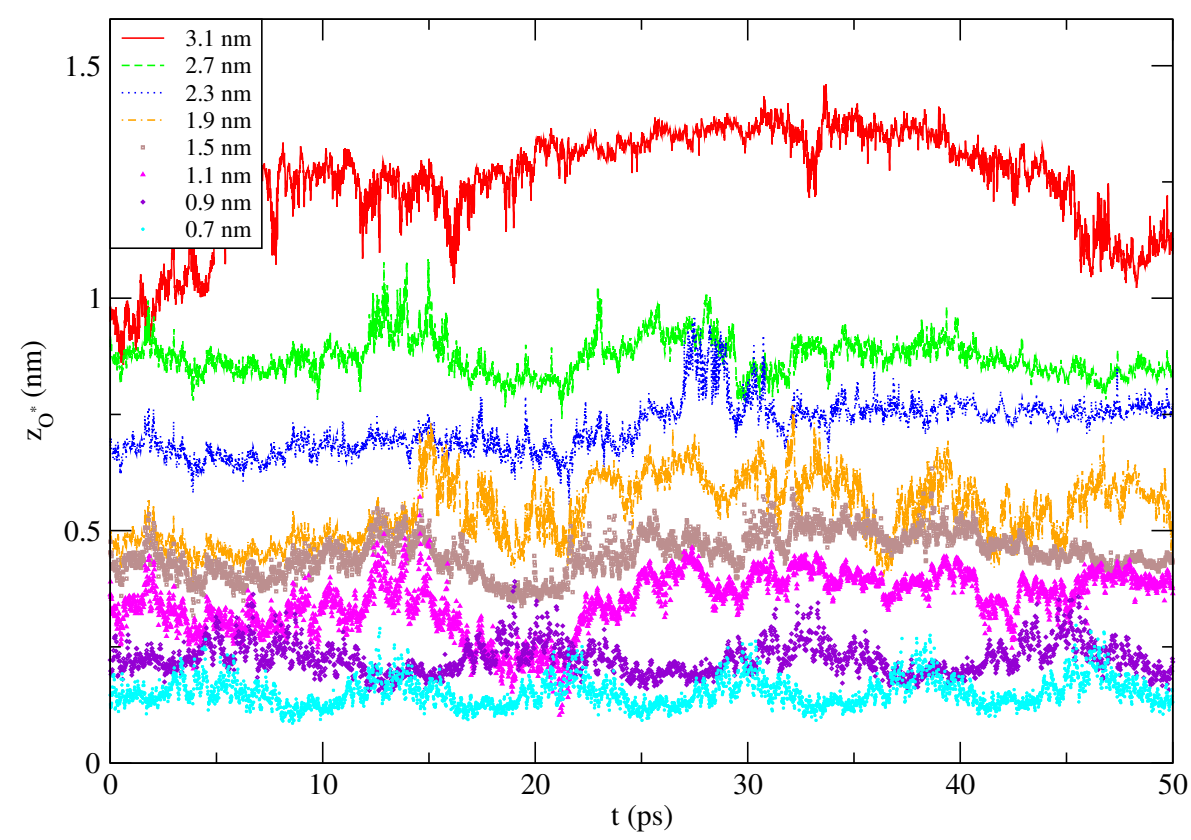

FIG. 2: (Color online) Oxygen pivot $\left(\mathrm{O}^{*}\right)$ z-position at different slab widths, between $d=3.1$ and $0.7 \mathrm{~nm}$. Here $z_{O}^{*}=0$ corresponds to the center of the slab. $d=3.1 \mathrm{~nm}$ (full red line); $d=2.7 \mathrm{~nm}$ (dashed green line); $d=2.3 \mathrm{~nm}$ (dotted blue line); $d=1.9 \mathrm{~nm}$ (dot-dashed orange line); $d=1.5 \mathrm{~nm}$ (brown squares); $d=1.1 \mathrm{~nm}$ (magenta triangles); $d=0.9 \mathrm{~nm}$ (violet diamonds); $d=0.7 \mathrm{~nm}$ (cyan stars). 

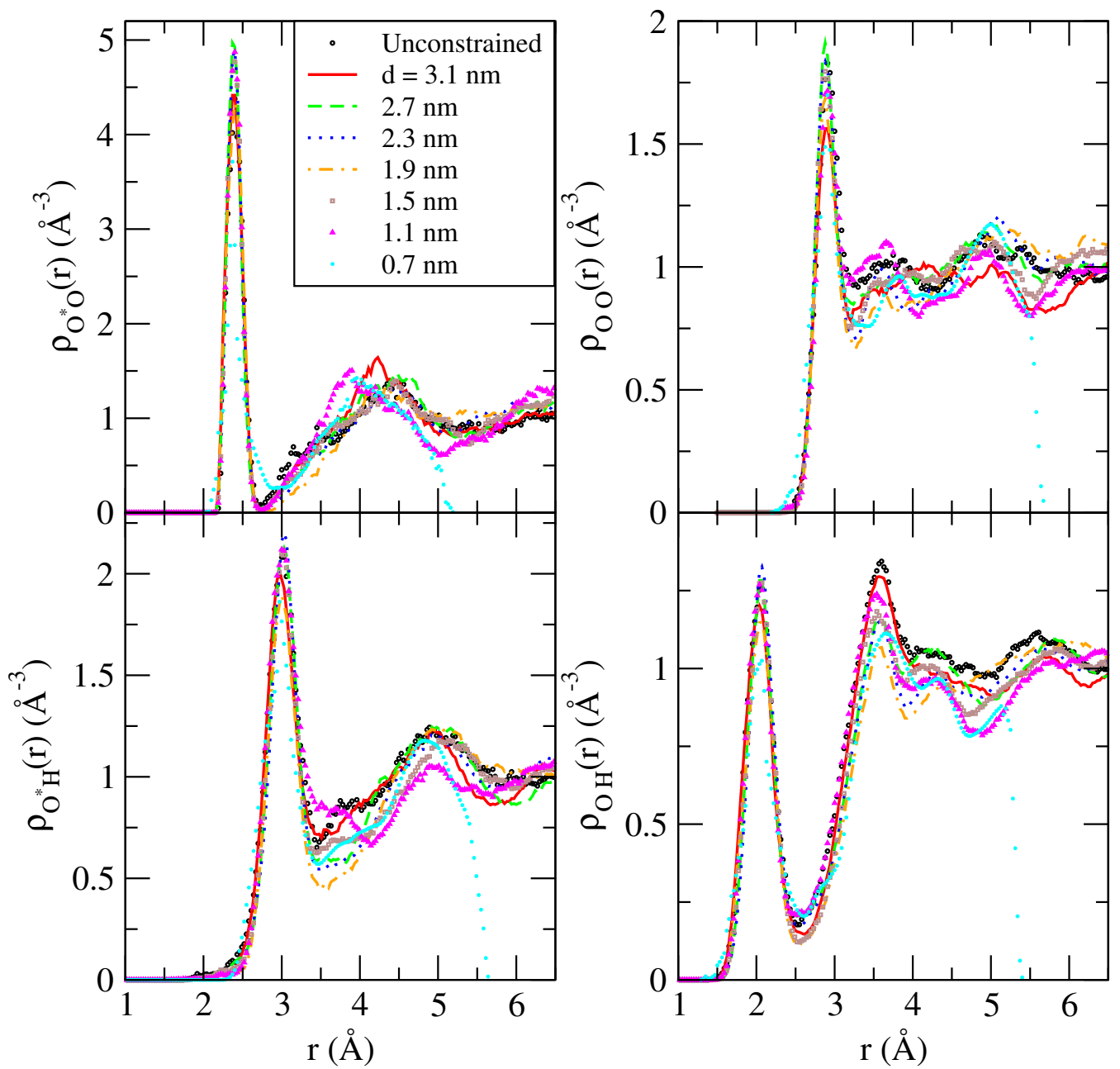

FIG. 3: (Color online) Oxygen pivot $\left(\mathrm{O}^{*}\right)$-oxygen solvent $(\mathrm{O})$ site-site pair correlation functions at different states (top left). Oxygen pivot $\left(\mathrm{O}^{*}\right)$-hydrogen solvent $(\mathrm{H})$ (bottom left). Oxygen-oxygen pair correlation functions (top right), Oxygen-hydrogen pair correlation functions (bottom right). Unconstrained water (black circles); $d=3.1 \mathrm{~nm}$ (full red line); $d=2.7 \mathrm{~nm}$ (dashed green line); $d=2.3$ $\mathrm{nm}$ (dotted blue line); $d=1.9 \mathrm{~nm}$ (dot-dashed orange line); $d=1.5 \mathrm{~nm}$ (brown squares); $d=1.1 \mathrm{~nm}$ (magenta triangles); $d=0.7 \mathrm{~nm}$ (cyan stars). 


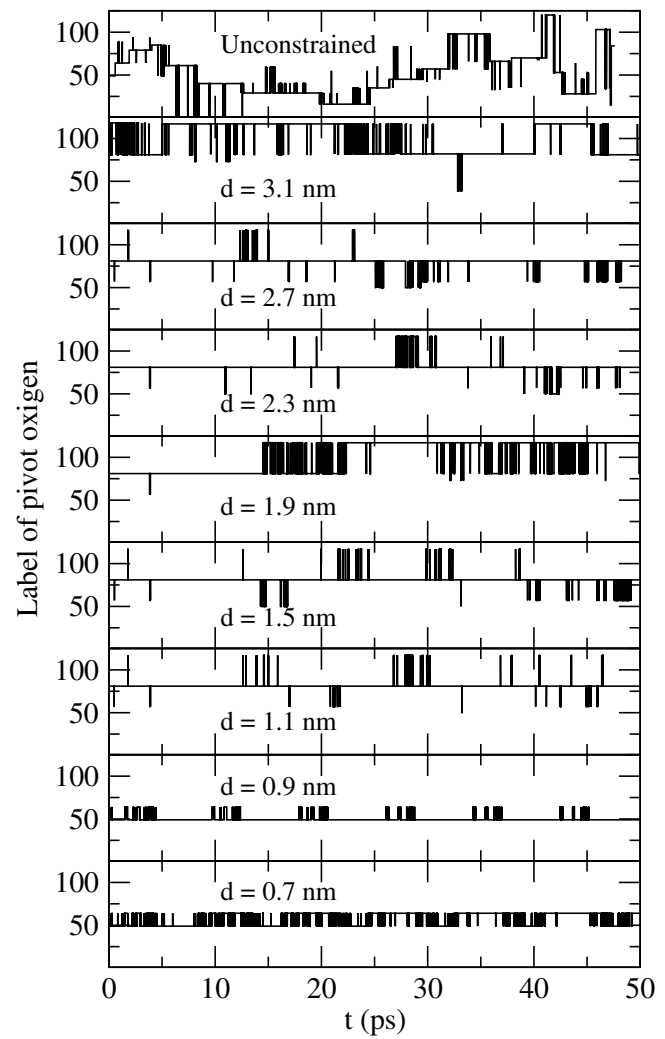

FIG. 4: Time evolution of pivot-oxygen labelling in different aqueous environments, from quasi 3D $(d=3.1 \mathrm{~nm})$ to quasi-2D water states $(d=0.7 \mathrm{~nm})$ (top to bottom). 


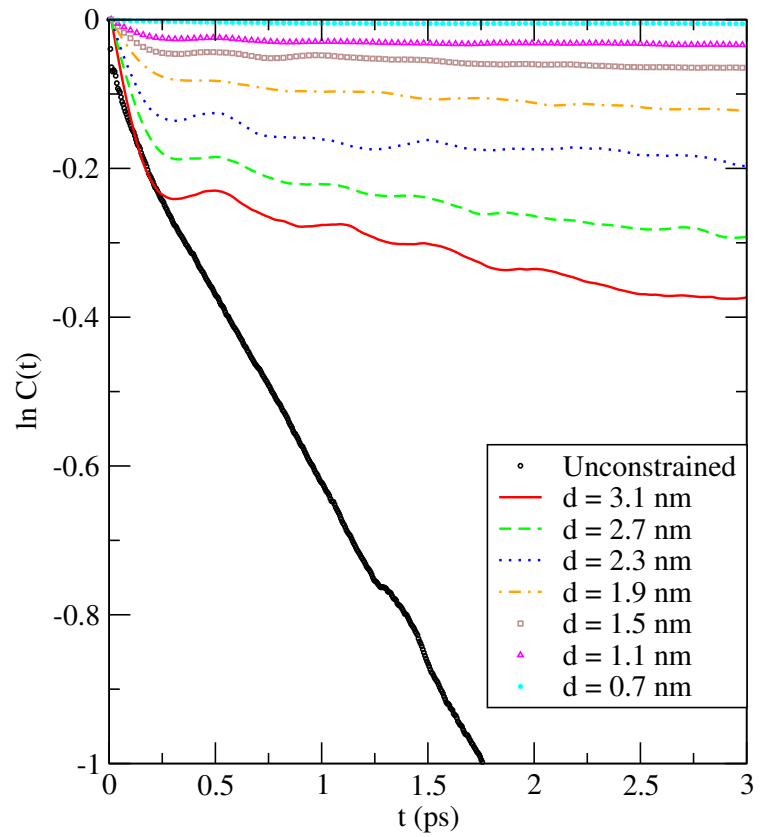

FIG. 5: (Color online) Logarithm of the population relaxations $C(t)$ for the pivot-oxygen label at different states. Unconstrained water (black circles); $d=3.1 \mathrm{~nm}$ (full red line); $d=2.7 \mathrm{~nm}$ (dashed green line); $d=2.3 \mathrm{~nm}$ (dotted blue line); $d=1.9 \mathrm{~nm}$ (dot-dashed orange line); $d=1.5 \mathrm{~nm}$ (brown squares); $d=1.1 \mathrm{~nm}$ (magenta triangles); $d=0.7 \mathrm{~nm}$ (cyan stars). 


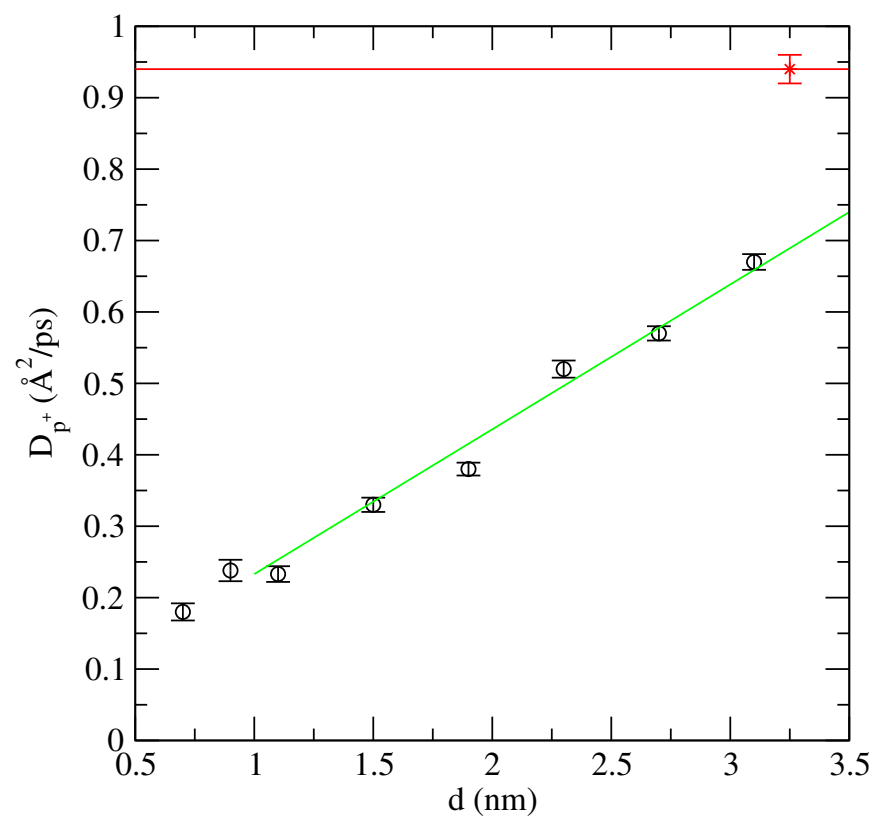

FIG. 6: (Color online) Diffusion coefficients of lone proton (circles) as a function of slab width d. Linear fit (green line) is indicated as an aid to the eye. The red star indicates the corresponding value for the unconstrained system. 


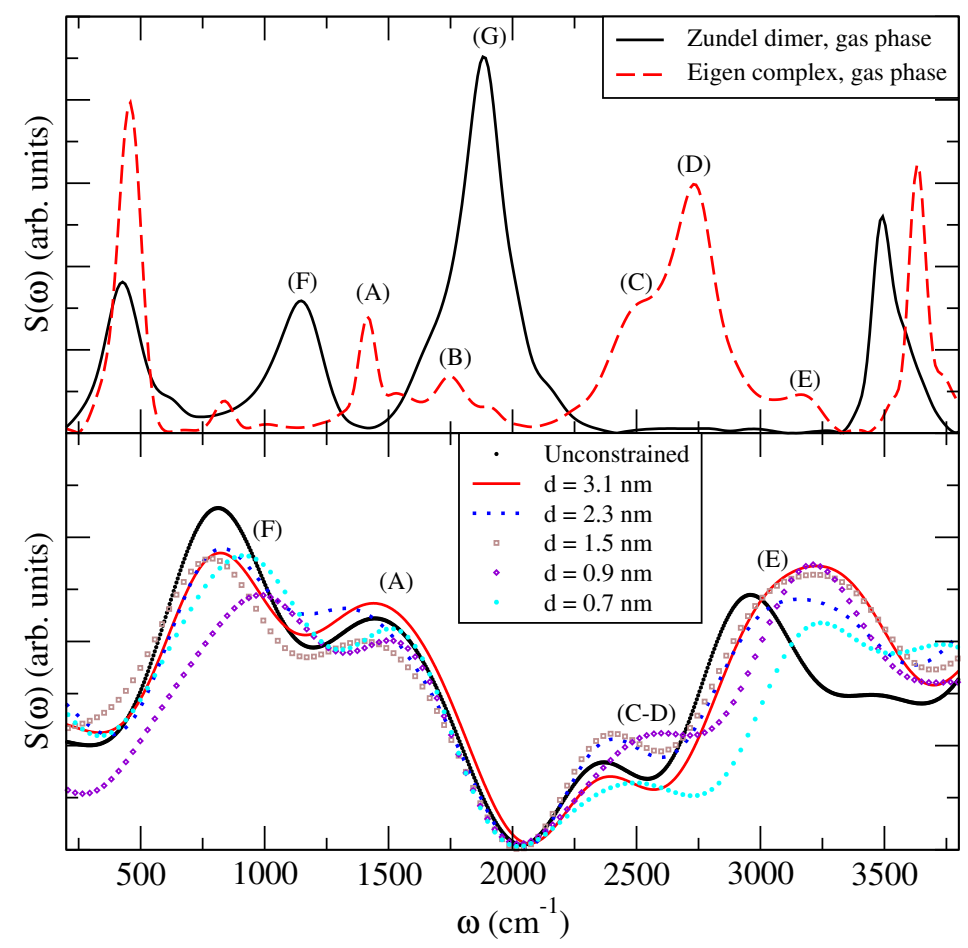

FIG. 7: (Color online) Vibrational densities of states $S_{p}^{+}(\omega)$ (in arbitrary units): proton in Zundel (black full line) and Eigen (red dashed line) complexes in gas phase at $298 \mathrm{~K}$ (top); confined states, from 3D water $(d=3.1 \mathrm{~nm})$ to $2 \mathrm{D}$ water states $(d=0.7 \mathrm{~nm})$ (bottom). Unconstrained water (black circles); $d=3.1 \mathrm{~nm}$ (full red line); $d=2.3 \mathrm{~nm}$ (dotted blue line); $d=1.5 \mathrm{~nm}$ (brown squares); $d=0.9 \mathrm{~nm}$ (violet diamonds); $d=0.7 \mathrm{~nm}$ (cyan stars). 